\title{
Utilidad del Ultrasonido en el diagnóstico de las hernias inguinales
}

\author{
Drs. Hansel Andreus $\boldsymbol{R}^{(1)}$, Lesly Solís $A^{(2)}$.
}

1. Especialista de 1er grado en Imagenología del Hospital Universitario "Dr. Carlos J Finlay", Ciudad de La Habana, Cuba.

2. Especialista de 1er grado en Imagenología y Profesora Asistente de Imagenología del Hospital Universitario

"Dr. Carlos J Finlay", Ciudad de La Habana, Cuba.

\section{Utility of us in the diagnosis of inguinal hernias}

Abstract: Objective. To determine the utility of ultrasound study in the diagnosis of inguinal hernias. Methods. Between March 2007 and March 2010, 283 patients aged over 16 years, with suspected inguinal hernia and inconclusive physical examination were studied. Preoperatively, patients underwent US examination of the involved area and findings were subsequently correlated with surgical outcomes. Results. Ultrasonographic and surgical diagnosis exhibited a coincidence rate of 94.7 per cent. Sensitivity and specificity were $96.7 \%$ and $81.6 \%$, respectively, as for general diagnosis of hernias; $94.4 \%$ and $98.4 \%$ for direct inguinal hernias; $95.6 \%$ and $96.8 \%$ for indirect hernias.

Key words: Inguinal hernia, Inguinal ultrasound, US diagnosis.

Resumen: Objetivos. Determinar la utilidad del ultrasonido en el diagnóstico de las hernias inguinales. Método. Entre marzo 2007 y marzo 2010 estudiamos 283 pacientes, mayores de 16 años, con sospecha de hernia inguinal y examen físico no concluyente a los que se les realizó ultrasonido de dicha región correlacionándolo con el diagnóstico quirúrgico. Resultados. El diagnóstico ultrasonográfico coincidió con el quirúrgico en el $94.7 \%$ de los casos. La sensibilidad y especificidad fue $96.7 \%$ y $81.6 \%$ para el diagnóstico general de hernias, $94.4 \%$ y $98.4 \%$ para las directas, $95.6 \%$ y $96.8 \%$ para las indirectas. Conclusiones. El estudio ultrasonográfico presenta un alto rendimiento en el diagnóstico de las hernias inguinales.

Palabras clave: Diagnóstico ultrasonográfico, Hernia inguinal, Ultrasonido inguinal.

Andreus H. Utilidad del Ultrasonido en el diagnóstico de las hernias inguinales. Rev Chil Radiol 2011; 17(2): 93-98. Correspondencia a: Dr. Hansel Andreus R. / vicky@ida.cu

Trabajo recibido el 03 de enero de 2011, aceptado para publicación el 20 de mayo de 2011.

\section{Introducción}

La hernia inguinal es una afección muy común siendo la más frecuente de todas las hernias abdominales, representando aproximadamente el $75 \%$ de las mismas. Toda hernia diagnosticada debe ser operada por el peligro de sufrir alguna complicación. Por lo general, el diagnóstico de la hernia inguinal es fácil de realizar y se basa fundamentalmente en el examen físico, sin embargo, en un número no despreciable de casos su diagnóstico clínico no es claro, especialmente en aquellos pacientes que sólo presentan dolor crónico o molestias poco precisas en esta zona, sin que se encuentren elementos al examen físico que justifiquen estos síntomas, los cuales son comunes en otras patologías de dicha región ${ }^{(1-6)}$. Por esto se hace necesario establecer un diagnóstico de certeza para definir la conducta a seguir. Pese a que los primeros usos del ultrasonido (US) en la rama de la medicina datan del año 1942 en la detección de tumores cerebrales ${ }^{(7)}$, es desde los comienzos de la década del $90^{(8,9)}$ que se emplea para el diagnóstico de la hernia inguinal. Existen muy pocas referencias en la literatura norteamericana acerca de su uso para el estudio la región inguinal, no siendo así en las referencias europeas. Sin embargo, son numerosos los trabajos que han demostrado su efectividad en el diagnóstico de esta patología ${ }^{(10-18)}$. En Cuba no está difundido el uso del US para evaluar dicha región, especialmente entre los cirujanos, quienes no reparan en este medio como un elemento que aporta una gran información sobre la anatomía de esta zona. Esto justifica la escasez de trabajos publicados sobre el tema, lo que motivó la realización de esta investigación, que tuvo como propósito determinar la utilidad del ultrasonido en el diagnóstico de las hernias inguinales, con comprobación quirúrgica. En este sentido nos planteamos como objetivo determinar si el ultrasonido permite, en 
nuestro medio, el diagnóstico certero de las hernias inguinales.

\section{Material y método}

Se realizó un estudio de evaluación de prueba diagnóstica desde marzo del año 2007 a marzo del año 2010 en 283 pacientes, mayores de 16 años, con sospecha de hernia inguinal y examen físico no concluyente, los cuales fueron explorados ecográficamente y posteriormente operados, aunque el ultrasonido preoperatorio resultara negativo, correlacionando los hallazgos quirúrgicos con el diagnóstico ultrasonográfico.

Los casos estudiados fueron pacientes provenientes de la consulta externa de cirugía y urología en el Hospital Universitario "Dr. Carlos J. Finlay". El estudio fue realizado en forma conjunta por un radiólogo y un cirujano, este último procedió a examinar la región inguinal de cada paciente, teniendo en cuenta los resultados de dicho examen; fue este especialista el que definió la indicación de la técnica ultrasonográfica, incluyéndose en el trabajo sólo aquellos casos que tuvieran un examen físico no concluyente o dudoso. Todos fueron examinados por el mismo cirujano. Excluimos dos pacientes que se negaron a operarse por ser imposible la comprobación quirúrgica.

Todos fueron sometidos a examen ultrasonográfico de ambas regiones inguinales (la sospechada y la contralateral) empleando un equipo ALOKA, modelo Pro Sound SSD-a5, con transductor lineal multifrecuencia de 7,5-13 Mhz, además dicho estudio se complementó con el Doppler color para identificar estructuras vasculares que sirvieron de referencia anatómica. La exploración ecográfica fue realizada en decúbito supino y de pie, sin y con maniobra de valsalva, cuya efectividad puede ser monitorizada por el aumento de calibre de la vena femoral. Esto se hizo con el fin de hacer una evaluación dinámica de la pared inguinal posterior y del anillo inguinal profundo (Figura 1a y b).

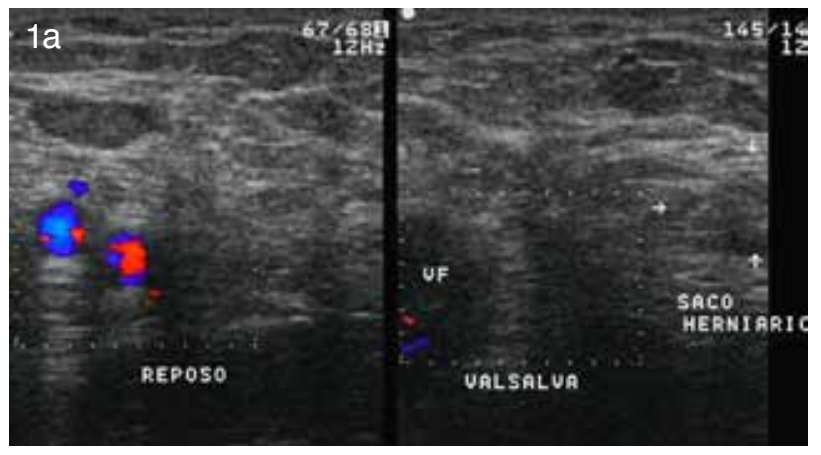

Figura 1. Evaluación dinámica de la pared inguinal posterior y del anillo inguinal profundo. a: Exploración en reposo y con maniobra de valsalva (MV). Se observa la vena femoral (VF) aumentada de calibre por MV, con flechas se señala el saco herniario visible después de aplicar dicha maniobra.

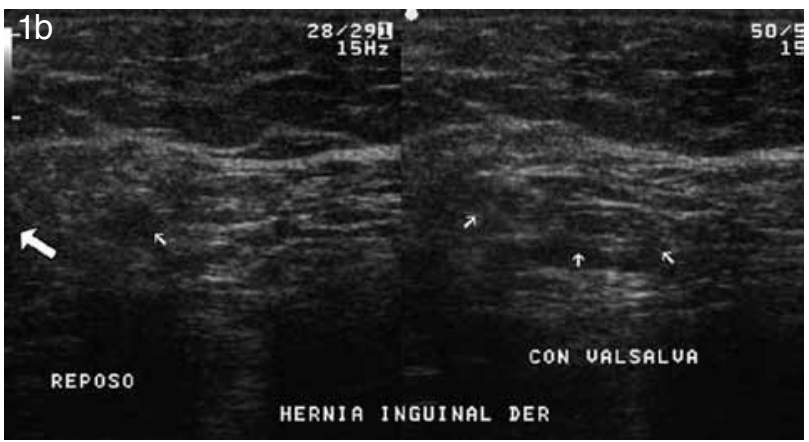

Figura 1b. Exploración en reposo y con maniobra de valsalva (MV). A través del anillo inguinal profundo (flecha gruesa) protruye el saco herniario con asas en su interior (flechas pequeñas) al realizar $M V$.

La evaluación se inició con cortes transversales para ubicar los vasos y delimitar los diferentes compartimentos, posteriormente se hicieron cortes oblicuos y longitudinales. Siempre se tomó la precaución de no provocar exceso de presión con el transductor, lo cual en ocasiones impide la visualización de pequeñas hernias. El uso de abundante gel elimina la necesidad de rasurar la región inguinal.

Se consideró como normal al canal inguinal, cuando aun, bajo las maniobras indicadas se observó un cierre adecuado del conducto inguinal así como una disminución de su diámetro cefalocaudal, y un anillo inguinal profundo normal, aquél cuyo diámetro no excediera los $4 \mathrm{~mm}$.

El método ecográfico de identificación de la hernia inguinal se basó en una serie de hallazgos dinámicos encontrados en la región, los cuales incluyeron ${ }^{(19)}$ :

1. Una dilatación del anillo inguinal profundo (anillo de más de $4 \mathrm{~mm}$ de diámetro o un incremento de 2 o más $\mathrm{mm}$ de su diámetro durante la maniobra de valsalva) dado por el desplazamiento de los vasos epigástricos profundos.

2. Protrusión de grasa o contenido peritoneal (asas, epiplón o líquido) a través del anillo inguinal profundo o de la pared inguinal posterior durante la maniobra de valsalva.

3. Balonamiento de la región inguinal cuando bajo la influencia de las maniobras señaladas se producía un incremento dinámico del diámetro de la pared inguinal posterior.

Una vez visualizados los reparos anatómicos (óseos y vasculares) y definidos los compartimentos se procedió a ubicar las hernias en ellos, lo que permitió diferenciarlas en directas e indirectas. Con este fin se identificaron las estructuras vasculares (Figura 2), especialmente la arteria epigástrica inferior que se ubica medial al anillo inguinal profundo. Con ello se determinó indirectamente la ubicación de este último, lo que hizo posible caracterizarlas, ya que las directas se ubican medial a ésta y las indirectas lateral a ella. La definición de los vasos femorales posibilitó delimitar la laguna 
muscular por fuera de ellos y naturalmente la laguna vascular que ellos ocupan. Además se caracterizó el contenido herniario, el cual casi siempre fue epiplón o asas intestinales que fueron fácilmente reconocidas por su peristalsis en tiempo real ${ }^{(17)}$ (Figuras 3 y 4 ).

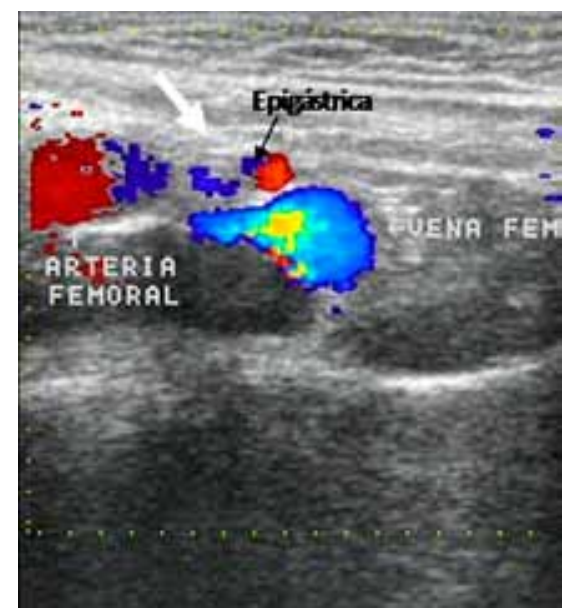

Figura 2. Anatomía ultrasonográfica del canal inguinal. Se identifican los vasos femorales con doppler color definiéndose la laguna vascular. Se demuestra la arteria epigástrica inferior y el sitio del anillo inguinal profundo con flecha blanca larga. La laguna muscular está lateral a la arteria.

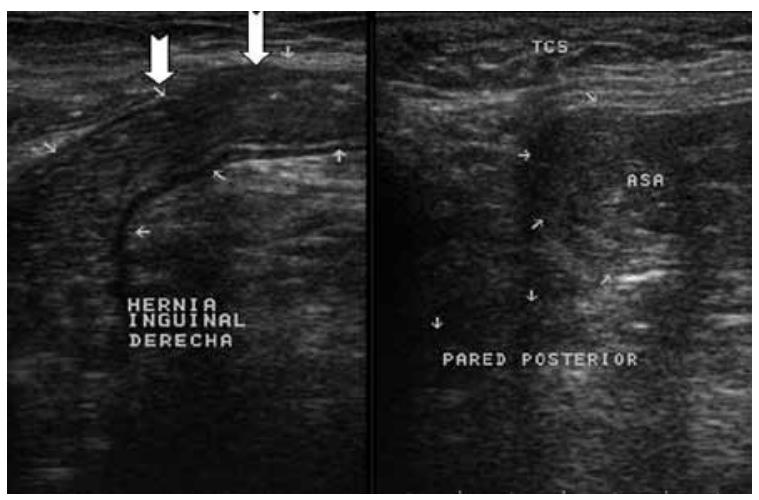

Figura 3. Hernia inguinal directa con contenido de asas intestinales. Cambio de morfología del contenido por peristalsis.

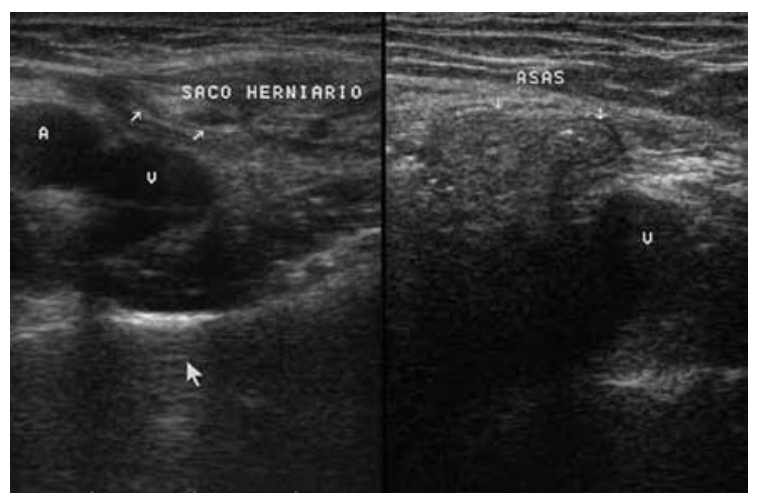

Figura 4. Hernia inguinal indirecta con contenido de asas intestinales. Se visualiza saco herniario protruyendo a través del anillo inguinal profundo (flechas pequeñas).
Los pacientes en los que el diagnóstico ultrasonográfico de hernia inguinal fue negativo también fueron explorados quirúrgicamente, previo consentimiento informado. En todos los casos se correlacionó el diagnóstico ultrasonográfico con el quirúrgico a partir del examen de dicha zona durante la intervención que fue realizada por el mismo cirujano que examinó al paciente.

Se utilizó la distribución de frecuencia calculando el porcentaje en variables cualitativas. En las variables cuantitativas se calculó la media aritmética. Se usó, además, el método del valor predictivo determinando sensibilidad y especificidad del ultrasonido utilizando como estándar de referencia los resultados quirúrgicos.

\section{Resultados}

Estudiamos 283 pacientes con sospecha de hernia inguinal y examen físico no concluyente, con edades comprendidas entre 17 y 78 años, con una media de 27,3 años, a los que se les realizó examen ultrasonográfico de ambas regiones inguinales y quirúrgico de la afecta; de ellos el grupo más afectado fue el de 20-29 años (36.7\%) seguido por el de 17-19 (22.3\%), perteneciendo el $85.9 \%$ al sexo masculino. La hernia inguinal indirecta fue la más observada $(55.1 \%)$, sin encontrarse diferencias significativas en cuanto al sexo. El aumento de volumen fue el motivo de consulta más común en los pacientes con hernia directa $(51.1 \%)$, mientras que en las indirectas predominó la asociación tumor-dolor (50.6\%). La región inguinal derecha fue la más afectada $(59.7 \%)$, independientemente del tipo de hernia. Ecográficamente la mayoría de los casos con hernia inguinal directa (69.3\%) presentaron protrusión de grasa o contenido peritoneal a través de la pared posterior. Sin embargo, la mayor parte de las indirectas (92\%) tuvo protrusión de grasa o contenido peritoneal a través del anillo inguinal profundo dilatado. Otros hallazgos demostrados con la evaluación ultrasonográfica fueron la presencia de adenopatías inguinales, de tumores del cordón y de quistes de Nuck. El diagnóstico ecográfico de hernia inguinal coincidió con el quirúrgico en el $94.7 \%$ de los casos, con una precisión del $96.6 \%$ para las directas, del $97.4 \%$ para las indirectas y del $79.5 \%$ para la ausencia de hernia. La aplicación del método de la determinación del valor predictivo nos brindó la siguiente información: (Tabla I, II y III).

\section{Discusión}

Nuestra certeza global, según coincidencia entre los hallazgos ultrasonográficos y los quirúrgicos, fue similar a la reportada por la mayoría de los autores que hacen referencia al tema ${ }^{(19,20)}$. La obtención de estos datos por ultrasonido facilita la intervención quirúrgica, ya que el cirujano sabe con anterioridad a lo que se enfrenta en el momento de la operación. 
Tabla I. Sensibilidad y especificidad del Ultrasonido en el diagnóstico de las hernias inguinales.

\begin{tabular}{|l|c|c|c|}
\hline \multirow{2}{*}{ Ultrasonido } & \multicolumn{2}{|c|}{ Cirugía } & \multirow{2}{*}{ Total } \\
\cline { 2 - 4 } & Positivos & Negativos & 244 \\
\hline $\begin{array}{l}\text { Positivos } \\
\text { Negativos }\end{array}$ & $837(\mathrm{VP})$ & $7(\mathrm{FP})$ & 39 \\
\hline Total & 245 & $31(\mathrm{FN})$ & 283 \\
\hline Sensibilidad (S): $96.7 \%$ & Especificidad (E): $81.6 \%$ \\
Valor predictivo positivo (VPP): $97.1 \%$ & Valor predictivo negativo (VPN): $79.4 \%$ \\
\hline
\end{tabular}

Tabla II. Sensibilidad y especificidad del Ultrasonido en el diagnóstico de las hernias inguinales directas.

\begin{tabular}{|l|c|c|c|}
\hline \multirow{2}{*}{ Ultrasonido } & \multicolumn{2}{|c|}{ Cirugía } & \multirow{2}{*}{ Total } \\
\cline { 2 - 4 } & Positivos & Negativos & 88 \\
\hline Positivo & $85(\mathrm{VP})$ & $3(\mathrm{FP})$ & 195 \\
Negativo & $5(\mathrm{FN})$ & $190(\mathrm{VN})$ & 283 \\
\hline Total & 90 & 193 & \multicolumn{2}{c|}{ Especificidad (E): $98.4 \%$} \\
\hline Sensibilidad (S): $94.4 \%$ & Valor predictivo negativo (VPN): $97.4 \%$ \\
Valor predictivo positivo (VPP): $96.6 \%$ &
\end{tabular}

Tabla III. Sensibilidad y especificidad del Ultrasonido en el diagnóstico de las hernias inguinales indirectas.

\begin{tabular}{|l|c|c|c|}
\hline \multirow{2}{*}{ Ultrasonido } & \multicolumn{2}{|c|}{ Cirugía } & \multirow{2}{*}{ Total } \\
\cline { 2 - 4 } & Positivos & Negativos \\
\hline Positivo & $152(\mathrm{VP})$ & $4(\mathrm{FP})$ & 156 \\
Negativo & $7(\mathrm{FN})$ & $120(\mathrm{VN})$ & 27 \\
\hline Total & 159 & 124 & 283 \\
\hline Sensibilidad (S): $95.6 \%$ & Especificidad (E): $96.8 \%$ \\
Valor predictivo positivo (VPP): $97.4 \%$ & Valor predictivo negativo (VPN): $94.5 \%$ \\
\hline
\end{tabular}

La certeza diagnóstica acorde a la coincidencia entre los hallazgos ultrasonográficos asociados y los quirúrgicos también resultó semejante a la descrita por el Dr. Solis( ${ }^{(20)}$ en nuestro centro; así como a la referida en la bibliografía internacional revisada ${ }^{(21,22-27)}$.

El nivel de coincidencia entre el diagnóstico ultrasonográfico y quirúrgico de hernia inguinal fue igual, incluso superior, a la mayoría de los estudios publicados en relación al tema, algunos de ellos se exponen en Tabla IV.

De los estudios revisados son pocos los que determinan la certeza considerando el tipo de hernia, entre ellos: Bradley $\mathrm{M}^{(31)}$ de inglaterra reportó, para las directas, valores de un $85.7 \%$, mientras que Babkova et al( ${ }^{(30)}$ de Rusia encontró cifras de un $71 \%$, para las indirectas Bradley $\mathrm{M}^{(31)}$ halló una certeza de un $97.3 \%$, en tanto que Babkova et al ${ }^{(30)}$ alcanzó un $84 \%$.

EI US mostró en general una alta sensibilidad y valor predictivo positivo, especificidad y valor predictivo negativo para el diagnóstico de las hernias inguinales, lo que significa que cuando un resultado ultrasonográfico sea positivo de hernia inguinal es altamente probable que el paciente la presente y al mismo tiempo si es negativo es muy probable que no la tenga. Estos resultados concuerdan con los publicados por otros autores Tabla V. 


\begin{tabular}{|c|c|c|c|}
\hline $\begin{array}{l}\text { Tabla IV. } \\
\text { Estudios }\end{array}$ & Año & $\begin{array}{c}\text { № de } \\
\text { Pacientes }\end{array}$ & $\begin{array}{c}\text { Certeza } \\
\%\end{array}$ \\
\hline Orchard JW'(28) & 1998 & 14 & 95,0 \\
\hline Van den Berg JC $\mathrm{JC}^{(29)}$ & 1999 & 41 & 81,5 \\
\hline Babkova IV ${ }^{(30)}$ & 2000 & 91 & 84,3 \\
\hline Lilli MC ${ }^{(22)}$ & 2002 & 41 & 92,0 \\
\hline Bradley $\mathrm{M}^{(31)}$ & 2004 & 118 & 95,0 \\
\hline Utria $^{(19)}$ & 2005 & 75 & 83,0 \\
\hline Robinson $\mathrm{P}^{(32)}$ & 2006 & 59 & 91,0 \\
\hline Solis ${ }^{(20)}$ & 2008 & 688 & 88,4 \\
\hline Autor & 2009 & 283 & 94,7 \\
\hline
\end{tabular}

Obtuvimos valores muy elevados de sensibilidad (94.4\%) y valor predictivo positivo (96.6\%), especificidad $(98.4 \%)$ y valor predictivo negativo $(97.4 \%)$ para el diagnóstico de las hernias directas, los que son semejantes a los reportados por otros autores como los que se mencionan en Tabla VI.

En Cuba no se encontraron estudios que analizaran por separado cada tipo de hernia inguinal.

También hallamos valores muy elevados de sensibilidad (95.6\%), valor predictivo positivo (97.4\%), especificidad $(96.8 \%)$ y valor predictivo negativo $(94.5 \%)$ para el diagnóstico de las hernias indirectas, los que son similares a los encontrados por otros investigadores Tabla VII.

Los errores del US en el diagnóstico de las hernias inguinales pueden deberse a la poca habilidad del operador. En nuestra investigación el examen fue realizado, en todos los casos, por el mismo especialista con experiencia de 7 años en la realización de dicho examen, evitando así la variabilidad interoperadores. Sin embargo, este estudio pudo estar sesgado al no medir la variabilidad intraoperador.

Aunque el US no es una técnica exacta en el diagnóstico de las hernias inguinales, las alternativas diagnósticas son menos precisas. Entre ellas están el examen físico $(E F)$, la herniografía y la resonancia magnética.

El examen físico posibilita un adecuado diagnóstico, pero en algunos casos puede determinar falsos positivos por la presencia de masas inguinales diferentes de hernias como lipomas preperitoneales o del cordón ${ }^{(29)}$, así como hidroceles. Sin embargo, el US permite fácilmente su diferenciación, además de hacer posible la identificación del contenido herniario y la exploración fisiológica del paciente ${ }^{(17)}$. En la serie de Chen y col. se demostró para la detección de hernia una seguridad de $97.9 \%$ para US y de $84 \%$ para el examen físico al ser comparados con cirugía ${ }^{(33)}$.

Robinson $\mathrm{P}^{(33)}$, halló una certeza de un $71 \%$ para el diagnóstico por herniografía, mientras que para US fue de un $91 \%$, lo que demuestra claramente la superioridad de la ecografía. Por otro lado, la herniografía es un examen que requiere más tiempo, uso de medios de contraste, así como de radiaciones ionizantes, además, el US permite definir mejor el contenido herniario, así como la exploración dinámica del paciente.

Van den Berg JC del Hospital St. Anthony en Holanda ${ }^{(28)}$ realizó un estudio en 41 pacientes encontrando una sensibilidad y especificidad del $74.5 \%$ y $96.3 \%$ respectivamente para el diagnóstico según examen físico, de $92.7 \%$ y $81.5 \%$ para el US y de $94.5 \%$ y la $96.35 \%$ para la resonancia. Este último método posee

\begin{tabular}{|llcccc|}
\hline $\begin{array}{l}\text { Tabla V. } \\
\text { Estudios }\end{array}$ & Año & $\begin{array}{c}\text { Sensb. } \\
\%\end{array}$ & $\begin{array}{c}\text { VPP } \\
\%\end{array}$ & $\begin{array}{c}\text { Espc. } \\
\%\end{array}$ & $\begin{array}{c}\text { VPN } \\
\%\end{array}$ \\
\hline Bradley M$^{(31)}$ & 2004 & 91.5 & 91.0 & 92.0 & 88.2 \\
Alam A $^{(26)}$ & 2005 & 65.0 & 67.2 & 62.0 & 60.7 \\
Robinson P(32) $_{\text {Solís(20) }}^{(2006}$ & 90.0 & 91.8 & 91.0 & 90.0 \\
\hline Autor & 2008 & 88.4 & 87.8 & 84.5 & 83.8 \\
\hline
\end{tabular}

\begin{tabular}{|lccc|}
\hline $\begin{array}{l}\text { Tabla VI. } \\
\text { Estudios }\end{array}$ & Año & $\begin{array}{c}\text { Sensibilidad } \\
\%\end{array}$ & $\begin{array}{c}\text { Especificidad } \\
\%\end{array}$ \\
\hline Kervancioglu(11) $^{(1)}$ & 2000 & 90,4 & 94,1 \\
Erez I(13) $^{(2002}$ & 93,2 & 95,0 \\
Bradley M $^{(31)}$ & 2004 & 86,0 & 97,0 \\
Robinson $\mathrm{P}^{(32)}$ & 2006 & 91,0 & 94,3 \\
\hline Autor & $\mathbf{2 0 0 9}$ & $\mathbf{9 4 , 4}$ & $\mathbf{9 8 , 4}$ \\
\hline
\end{tabular}

\begin{tabular}{|lccc|}
\hline $\begin{array}{l}\text { Tabla VII. } \\
\text { Estudios }\end{array}$ & Año & $\begin{array}{c}\text { Sensibilidad } \\
\%\end{array}$ & $\begin{array}{c}\text { Especificidad } \\
\%\end{array}$ \\
\hline Kervancioglu(11) $^{(11)}$ & 2000 & 95,0 & 91,4 \\
Erez I(13) $^{\text {Bradley M(31) }}$ & 2002 & 92,6 & 88,4 \\
Robinson $\mathrm{P}^{(32)}$ & 2004 & 97,0 & 87,0 \\
\hline Autor & 2006 & 93,0 & 89,7 \\
\hline
\end{tabular}


mayor efectividad, pero es mucho más costoso, no está disponible en todas las instituciones de salud, presenta algunas contraindicaciones para su uso y el tiempo de realización es prolongado.

En sentido general nuestros resultados y los de los numerosos autores mencionados en este trabajo nos permiten asegurar que el US es un método eficiente para diagnosticar las hernias inguinales.

\section{Conclusiones}

El ultrasonido es un método eficiente para diagnosticar las hernias inguinales, permitiendo además su caracterización.

\section{Bibliografía}

1. Mirilas P, Colborn GL, McClusky DA. The history of anatomy and surgery of the preperitoneal space. Arch Surg 2005; 140(1): 90-94.

2. Stoppa R, Wantz G, Munegato G. Hernia healers: an illustrated history. Hardcover. ed. Michigan University: Arnette; 1998. 175 p.

3. Hair A, Paterson C, Wright D, Baxter JN, O'Dwyer PJ. What effects does the duration of an inguinal hernia have on patients symptoms? J Am Coll Surg 2005; 193: 125-129.

4. Shadbolt CL, Heinze SB, Dietrich RB. Imaging of groin masses: Inguinal anatomy and pathologic conditions revisited. Radiographics 2005; 21: S261-S271.

5. Ekberg O, Persson NH, Abrahamsson P. Long standing groin pain in athletes: a multidisciplinary approach. Sport med 1998; 6(1): 56-61.

6. Estwanick JJ, Sloane B, Rosenberg MA. Groin strain and other possible causes of groin pain. Phys Sportsmed 1990; 18 (2): 54-65.

7. Gonzalo E. Díaz M. Ultrasonido [Internet]. Bogotá; [updated 2005 Feb 2; cited 2006 Mar 24]. Available from: http://www.drgdiaz.com/eco/ultrasonido/ultrasonidos/ ultrasonido.shtml.

8. May E, O'Brien A, Liendo C., y col. Metalosis por titanio. Diagnóstico diferencial de masa del compartimento iliopsoas. Caso clínico. Rev Chil Radiol 2002; 8: 182184.

9. Erez I, Kovalivker M, Schneider N. et al. Elective sonographic evaluation of inguinal hernias in children. An effective alternative to routine contralateral exploration. Pediatr Surg 1993; 8: 415-418.

10. Chen KC, Chu CC, Chou TY, Wu CJ. Ultrasonography for inguinal hernias in boys. J Pediatr Surg 1998; 33(12): 1784-1787.

11. Kervancioglu R, Baryman MM. Ultrasonographic evaluation of bilateral groins with unilateral inguinal hernia. Acta Radiol 2000; 41 (6): 653-657.

12. Lawrenz K, Hollman AS, Carachi R, Cacciaguerra S. Ultrasound assessment of the contralateral groin with unilateral inguinal hernia. Clin Radiol 2004; 49: 546548.

13. Erez I, Rathause V. Preoperative ultrasound and intraoperative findings of inguinal hernias: a prospective study of 642 patient. J Surg 2002; 37(6): 865-868.

14. Graf JL, Caty MG, Martin DJ, Glick PL. Pediatric hernias. Semin Ultrasound CT MR 2002; 23(2): 197-200.
15. Zhang GQ, Sugiyama M, Hagi H, Urata T, Shimamori N, Atomi Y. Groin hernias in adults: value of color Doppler sonography in their classification. J Clin Ultrasound 2001; 29(8): 429-434.

16. Jamadar DA, Jacobson JA, Morag Y, Girish G, Dong $\mathrm{Q}, \mathrm{Al}-$ Hawary $\mathrm{M}$ et al. Characteristic Locations of inguinal region and anterior abdominal wall hernias: Sonographic Appearances and identification of clinical pitfalls. Am J Roentgenol 2007; 188: 1356-1364.

17. Arce JD. Región inguinal: ultrasonografía. Rev Chil Radiol 2004; 10: 58-69.

18. Korenkov M, Paul A, Troidl H. Color duplex sonography: diagnostic tool in the differentiation of inguinal hernias. J Ultrasound Med 1999; 18: 565-568.

19. Utria Velásquez A. La ecografía en el diagnóstico de la hernia inguinal. [Tesis de especialidad]. Instituto Superior de Ciencias médicas de La Habana 2005.

20. Solis Chávez SL. La ecografía en el diagnóstico de las enfermedades de la región inguinal y femoral. [Tesis doctoral]. Instituto Superior de Ciencias médicas de La Habana 2008.

21. Heller CA, Marucci DD, Dunn T. Inguinal canal lipoma. Clin Anat 2004; 15(4): 280-285.

22. Lilly MC, Arregui ME. Tumors of the cord and round ligament. Ann Surg 2002; 235(4): 586-590.

23. Yang DM, Kim HC, Lim JW, Jim W, Ryu CW, Kim GY, et al. Sonographic findings of groin masses. J Ultasound Med 2007; 26(5): 605-614.

24. Safak AA, Erdogmus B, Yazici B, Gokgoz AT. Hidrocele of the canal of Nuck: Sonographic and MRI appearances. J Clin Ultrasound 2007; 35(9): 531-532.

25. Stickel WH, Manner M. Female hidrocele (cyst of the canal of Nuck): sonographic appereance of a rare and little-known disorder. Surg Endosc 2006 ; 17(12): 202204.

26. Alam A, Nice C, Uberoi R. The accuracy of ultrasound in the diagnosis of clinically occult groin hernias in adults. Eur Radiol 2005; 15(12): 2457-2461.

27. Hata S, Takahashi Y, Nakamura T, Suzuki R, Kitada M, Shimano T. Preoperative sonographic evaluation is a useful method of detecting contralateral patent processus vaginalis in pediatric patients with unilateral inguinal hernia. J Pediatr Surg 2004; 39: 1396-1399.

28. Orchard JW, Read JW, Neophyton J, Garlick D. Groin pain associated with ultrasound finding of inguinal canal posterior wall deficiency in Australian Rules footballers. Br J Sports Med. 1998; 32(2): 134-139.

29. Van den Berg JC, de Valois JC, Go PM et al. Detection of groin hernia with physical examination, ultrasound and MRI compared with laparoscopic findings. Invest Radiol 1999; 34: 739-743.

30. Babkova IV, Bozhko VV. Ultrasound assessment in diagnosis of uncomplicated inguinal hernia. Khirurgiia 2000; 2: 46-50.

31. Bradley M, Morgan D. The groin hernia - an untrasound diagnosis? Ann R Coll Surg 2004; 85(3): 178-180.

32. Robinson P, Hensor E, Lansdown MJ, Ambrose NS, Chapman $\mathrm{AH}$. Inguinofemoral hernia: accuracy of sonography in patients with indeterminate clinical features. Am J Roentgenol 2006; 187(5): 1168-1178.

33. Chen KC, Chu CC, Chou TY, Wu CJ. Ultrasonography for inguinal hernias in boys. J Pediatr Surg 1998; 33: 1784-1787. 